\title{
Empfehlungen für die Dokumentation von Ethik-Fallberatungen
}

\author{
AG ,Ethikberatung im Krankenhaus“ in der Akademie für Ethik \\ in der Medizin e. V. (AEM) - Uwe Fahr • Beate Herrmann • \\ Arnd T. May $\cdot$ Antje Reinhardt-Gilmour $\cdot$ Eva C. Winkler
}

Online publiziert: 27. August 2010

(C) Die Autor(en) 2010. Dieser Artikel ist auf Springerlink.com mit Open Access verfügbar.

\section{Einleitung}

Die Zentrale Ethikkommission bei der Bundesärztekammer fordert in ihrer Stellungnahme zur Ethikberatung in der klinischen Medizin, dass Ethikberatungen schriftlich für die Krankenunterlagen dokumentiert werden [9]. Die hier vorliegenden Empfehlungen geben Hin-

Die Empfehlungen wurden von der Arbeitsgruppe „Ethikberatung im Krankenhaus“ in der Akademie für Ethik in der Medizin e. V. (AEM) erarbeitet. Mitglieder der Arbeitsgruppe waren: S. Dinges (Wien), A. Dörries (Hannover), G. Greif-Higer (Mainz), M. Herberhold (Altena), M. Kettner (Witten), K. Kobert (Bielefeld), G. Neitzke (Hannover), T. Peters (Bochum), M. Pfäfflin (Bielefeld), S. Reiter-Theil (Basel), G. Richter (Marburg), A. Riedel (Esslingen), K. Schmidt (Frankfurt/M.), A. Simon (Göttingen), J. Vollmann (Bochum). Arbeitsgruppen in der AEM sind offene Foren für den Austausch unterschiedlicher Standpunkte und Positionen. Der Inhalt der von ihnen veröffentlichen Beiträge wird allein von den genannten Autorinnen und Autoren verantwortet. Er repräsentiert nicht notwendigerweise die Meinung der AEM oder ihrer Organe.

AG „Ethikberatung im Krankenhaus“ in der Akademie für Ethik in der Medizin e. V. (AEM) ( $\bowtie$ ) Humboldtallee 36, 37073 Göttingen, Deutschland

E-Mail: ag-ethikberatung@ethikkomitee.de

U. Fahr

Ethikberatung \& Coaching, Erlangen, Deutschland

B. Herrmann

Medizinische Klinik, Universitätsklinik, Heidelberg, Deutschland

A. T. May

EthikZentrum.de, Zentrum für Angewandte Ethik, Recklinghausen, Deutschland
A. Reinhardt-Gilmour
Ilmenau, Deutschland
E. C. Winkler
Medizinische Klinik und Poliklinik III, Klinikum Großhadern, München, Deutschland 
weise, wie dies geschehen kann. Sie richten sich vornehmlich an Mitarbeiter ${ }^{1}$ in Klinischen Ethikkomitees und Arbeitsgruppen, die Ethik-Fallberatung anbieten. Sie geben Orientierung, welche Themen und Aspekte bei einer Dokumentation zu berücksichtigen sind.

Da gegenwärtig unterschiedliche Konzepte der Dokumentation diskutiert und praktiziert werden [6], müssen die Beratenden über die Form der Ethik-Fallberatung entscheiden, die sie ihrer Tätigkeit zugrunde legen [2, 4]. Im Einzelfall kann dies Auswirkungen auf die Art der Dokumentation haben, die hier nicht berücksichtigt werden.

Bei der Dokumentation von Ethik-Fallberatungen ist zwischen einer externen Dokumentation (Ergebnisberichte) und der internen Dokumentation (Aufzeichnungen des Beraters) zu unterscheiden [5]. Die hier vorliegenden Empfehlungen geben Hinweise zu den Ergebnisberichten. Andere Formen der Dokumentation werden hier nicht erläutert.

\section{Grundlagen}

2.1 Ethik-Fallberatungen: Als Ethik-Fallberatungen werden Besprechungen verstanden, bei denen ein konkreter Behandlungsfall unter ethischen Gesichtspunkten diskutiert wird. Diese Besprechung ist prospektiv, d. h. sie soll dabei helfen, ein gemeinsam getragenes Behandlungskonzept festzulegen, verschiedene Behandlungsoptionen in ihren Konsequenzen und Gründen zu erörtern, ethisch zu bewerten und bei der Festlegung einer Behandlungsoption zu helfen. Sie wird in der Regel auf der Station stattfinden, auf welcher der Patient behandelt wird, bzw. in der Wohngruppe des Bewohners in der Altenhilfe.

2.2 Konsensorientierung der Ethikberatung: Im Idealfall wird im Rahmen dieser Beratung ein Konsens erarbeitet, der in der Dokumentation dargelegt wird. Dieser Konsens hat den Charakter einer Empfehlung. Er entbindet die Beteiligten jedoch nicht von ihrer jeweiligen Verantwortung. In der Beratung können verschiedene Handlungswege in Abhängigkeit von der weiteren Entwicklung bezüglich medizinischer, pflegerischer oder anderer Aspekte erörtert oder ein Katalog von moralisch empfehlenswerten Maßnahmen entwickelt werden. Berücksichtigung sollte auch die Frage finden, ob alle Entscheidungsträger an der Besprechung teilnehmen konnten. Ist dies nicht der Fall, sollten die Handlungsmöglichkeiten unter moralischen Gesichtspunkten erörtert werden, um diese später mit den fehlenden Entscheidungsträgern zu diskutieren.

2.3 Schutz von Beratungen: Ethik-Fallberatung findet in einem Spannungsfeld zwischen den moralisch-praktischen Diskursen, rechtlichen Vorgaben und persönlichen Wertüberzeugungen statt und braucht einen besonderen Vertrauensschutz. Dokumentationen sollten daher so abgefasst werden, dass einerseits wahrheitsgemäß die Ergebnisse der Beratung dargelegt werden, andererseits ein offener und aufrichtiger Beratungsprozess dadurch nicht gefährdet wird.

2.4 Ratsuchende: Als Ratsuchende werden im Folgenden alle Personen verstanden, die in einer anderen Funktion als der des Ethikberaters an einer Ethik-Einzelfallberatung teilnehmen. Dies können u. a. Ärzte, Pflegende, Patienten/Bewohner, Bevollmächtigte, gesetzliche Betreuer und Angehörige sein.

\footnotetext{
${ }^{1}$ Auf die Verwendung beider Geschlechtsformen wird lediglich mit Blick auf die bessere Lesbarkeit des Textes verzichtet.
} 


\section{Ziele der Dokumentation}

3.1 Orientierung und Erinnerungsfunktion: Dokumentationen können den Ratsuchenden Orientierung, Erinnerungshilfe und Erleichterung bei der schriftlichen Niederlegung der Fallberatung in der Krankenakte sein. Die schriftliche Dokumentation in der Patientenakte ermöglicht es, dass das gesamte Behandlungsteam über die weitere Vorgehensweise informiert ist.

3.2 Absicherung: Die Dokumentation erhöht die Entscheidungssicherheit der Entscheidungsträger. Ethikberater tragen zwar keine Verantwortung für die Entscheidungen von Ärzten, Pflegenden oder Betreuern, Bevollmächtigten - sie tragen jedoch Verantwortung für die angemessene Durchführung der Beratung. Die Dokumentation legt dar, dass eine Ethik-Fallberatung gemäß den Standards (AEM, 2010) durchgeführt wurde [1].

3.3 Qualitätssicherung: Eine Aufgabe des Ethikberaters ist die Qualitätssicherung des Beratungsprozesses. Die Dokumentation der Ethik-Fallberatung ist ein Merkmal der Prozess- und Ergebnisqualität und die Grundlage für weiterführende Evaluation [7].

3.4 Ausbildung von Ethikberatern: Im Rahmen der Ausbildung von Ethikberatern können (anonymisierte) Dokumentationen von Beratungsprozessen herangezogen und mit erfahrenen Kollegen durchgesprochen werden [8].

3.5 Tätigkeitsnachweis: Dokumentationen von Fallberatungen stellen eine Grundlage für Tätigkeitsnachweise im Rahmen von Geschäftsberichten oder Jahresberichten dar.

\section{Ergebnisberichte als Dokumentation einer Ethik-Fallberatung}

Das Ergebnis einer Ethik-Fallberatung einschließlich einer Begründung ist in Form eines Ergebnisberichts durch den Ethikberater zu dokumentieren.

Inhalte: Der Ergebnisbericht enthält

- Datum, Ort und Dauer der Beratung,

- Namen und Funktion der Teilnehmer,

- ethische Fragestellung(en),

- aktuelle medizinische, pflegerische und psychosoziale Situation,

- Wünsche und Wertvorstellungen, erklärter bzw. mutmaßlicher Wille des Patienten,

- Ergebnis der Beratung (erzielter Konsens oder divergierende Meinungen) einschließlich Begründung.

4.1 Vertraulichkeit: Ergebnisberichte sind Bestandteile von Krankenunterlagen bzw. der Bewohnerdokumentation in der Altenhilfe. Als solche wenden sie sich an alle, die an der Behandlung/Versorgung beteiligt sind und entsprechende Einsichtsrechte in die jeweiligen Unterlagen haben [3]. Daher ist die Vertraulichkeit der Beratungssituation zu wahren. Generell sollte auf einzelne Zitate, die bestimmten Teilnehmern zugeschrieben werden können, eher verzichtet werden, es sei denn, dass diese ausdrücklich zustimmen. Eine wichtige Ausnahme stellen Äußerungen über den mutmaßlichen Patientenwillen dar. Diese Person sollte ausdrücklich genannt werden, wenn sie zitiert wird. Ihr sollte die Möglichkeit zur Korrektur eingeräumt werden.

4.2 Dokumentationspflicht: Im Interesse der Patienten, der Ratsuchenden und der Akzeptanz von Ethikberatung wird Ethikberatern empfohlen, die Dokumentation von Beratungen selbst zu übernehmen. Ergebnisse müssen in jedem Fall dokumentiert werden, wenn sie Konsequenzen für die Behandlung/Versorgung des Patienten oder Bewohners 
haben. Zu Beginn der Beratung sollte vereinbart werden, wer die Dokumentation in die Unterlagen einfügt.

4.3 Zustimmung der Beteiligten: Den an der Beratung Beteiligten sollte der Ergebnisbericht vor Einfügen in die Krankenunterlagen oder der Bewohnerdokumentation zur Kenntnis gegeben werden.

4.4 Unterschrift: Es wird empfohlen, dass der Ergebnisbericht durch den Protokollanten unterschrieben wird. Ergänzend kann der Ergebnisbericht von mehreren oder allen beteiligten Personen unterzeichnet werden. Es besteht die Möglichkeit, bereits während der Beratung die wesentlichen Ergebnisse in einem kurzen Formblatt zu dokumentieren, dieses am Ende der Beratung vorzulesen und mit der Bemerkung ,,vorgelesen und bestätigt" zu versehen.

4.5 Dokumentationsbögen: Gegliederte Erhebungsbögen („Dokumentationsbögen“) können sinnvoll sein, da sie sowohl eine Arbeitserleichterung als auch eine Strukturierungshilfe für den Beratungsprozess sein können. Sie sollten jedoch nicht die Aufbereitung der Ergebnisse einer Ethik-Fallberatung in einem Ergebnisbericht ersetzen.

\section{Aufbewahrung, Datenschutz und Einsichtsrecht}

5.1 Schweigepflicht und datenschutzrechtliche Vorgaben: Für jede Form der Dokumentation gelten die Regelungen des Datenschutzgesetzes sowie andere einschlägige rechtliche Regelungen. Der Ethikberater hat über die Tatsache der Beratung sowie deren Inhalte Schweigen zu wahren und sollte auf die Einhaltung der Schweigepflicht vor Beginn der Beratung hinweisen.

5.2 Aufbewahrung: Sofern Ergebnisberichte auch außerhalb der Patientenakte durch die Ethikberater aufbewahrt werden, hat dies gemäß datenschutzrechtlichen Vorgaben zu geschehen. Besondere Sorgfalt ist dabei auf die Verwahrung von Unterlagen auf elektronischen Datenträgern oder anderen Speichermedien zu verwenden. Die Dokumentationen sollten wie ärztliche Aufzeichnungen für die Dauer von zehn Jahren aufbewahrt werden. Es wird empfohlen, handschriftliche Notizen u.ä. nach Abschluss der Beratung zu vernichten.

5.3 Einsichtsrechte: Ratsuchende sowie die betroffenen Patienten oder Bewohner bzw. Betreuer/Bevollmächtigte haben das Recht zur Einsicht in den Ergebnisbericht.

5.4 Verlegung des Patienten: Im Rahmen der Verlegung des Patienten kann im Arztbrief auf wichtige Ergebnisse der Ethikberatung eingegangen werden. Sollte ein Ergebnisbericht weitergereicht werden, so ist dieser mit den Worten zu versehen: „Dieser Bericht ist Bestandteil der Patientenakte und vertraulich zu behandeln.“

Open Access Dieser Artikel unterliegt den Bedingungen der Creative Commons Attribution Noncommercial License. Dadurch sind die nichtkommerzielle Nutzung, Verteilung und Reproduktion erlaubt, sofern der/die Originalautor/en und die Quelle angegeben sind.

\section{Literatur}

1. Akademie für Ethik in der Medizin e.V. (2010) Standards für Ethikberatung in Einrichtungen des Gesundheitswesens. Ethik Med 22:149-153

2. Aulisio MP, Arnold RM, Youngner SJ (2003) Ethics consultation. From theory to practice. John Hopkins University Press, Baltimore 
3. Deutsch E, Spickhoff A (2008) Medizinrecht: Arztrecht, Arzneimittelrecht, Medizinproduktegesetz und Transfusionsrecht, 6. Aufl. Springer, Berlin

4. Dörries A, Neitzke G, Simon A, Vollman J (2008) Klinische Ethikberatung. Ein Praxisbuch für Krankenhäuser und Einrichtungen der Altenpflege, 2. überarb.Aufl. Kohlhammer, Stuttgart

5. Fahr U (2009) Die Dokumentation Klinischer Ethikberatung. Ethik Med 21:32-44

6. Freedman B, Weijer C, Bereza E (1993) Case notes and charting of bioethical case consultation. HEC Forum 5:176-195

7. Kobert K, Pfafflin M, Reiter-Theil S (2008) Evaluation des klinischen Ethik-Beratungsdienstes. Hintergrund, Konzepte und Strategie im Ev. Krankenhaus Bielefeld. Ethik Med 20:122-133

8. Simon A, May AT, Neitzke G (2005) Curriculum „Ethikberatung im Krankenhaus“. Ethik Med $17: 322-326$

9. Zentrale Ethikkommission (2006) Stellungnahme der Zentralen Kommission zur Wahrung ethischer Grundsätze in der Medizin und ihren Grenzgebieten (Zentrale Ethikkommission) bei der Bundesärztekammer zur Ethikberatung in der Klinischen Medizin (24. Januar 2006). Dtsch Arztebl 103: A1703-A1707 